\title{
Dynamics of fibroblast activation in the infarcted heart
}

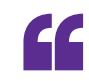

are that better

molecular

characterization

of the specific

stages of

fibroblast

differentiation

... will permit

better

targeted

therapies to

control these

cells in the

heart

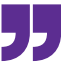

\section{Our hopes}

After myocardial infarction (MI), which causes a substantial loss of cardiomyocytes, fibroblasts are activated, proliferate, and differentiate into extracellular matrix (ECM)producing myofibroblasts that contribute to the formation of a fibrotic scar in the wounded area. In the non-regenerative heart, the scar persists, stabilized by fibroblasts that revert to a quiescent state. A new study provides further insights into the phenotypic changes of cardiac fibroblasts during scar formation. "Fibroblasts are absolutely critical for acute healing of the heart after injury," explains lead study investigator Jeffery Molkentin, "yet at the same time, overzealous long-term activation of fibroblasts in more chronic heart disease states can hasten the demise of the heart." Molkentin remarks that studies into heart diseases have now started to focus on fibroblasts, with the recent advent of new genetic tools to investigate this cell type.

In this study, Molkentin and colleagues used three inducible, lineage-tracing mouse models, Tcf21-MerCreMer, Postn-MerCreMer, and Acta2-CreER, to reliably

identify fibroblast dynamics

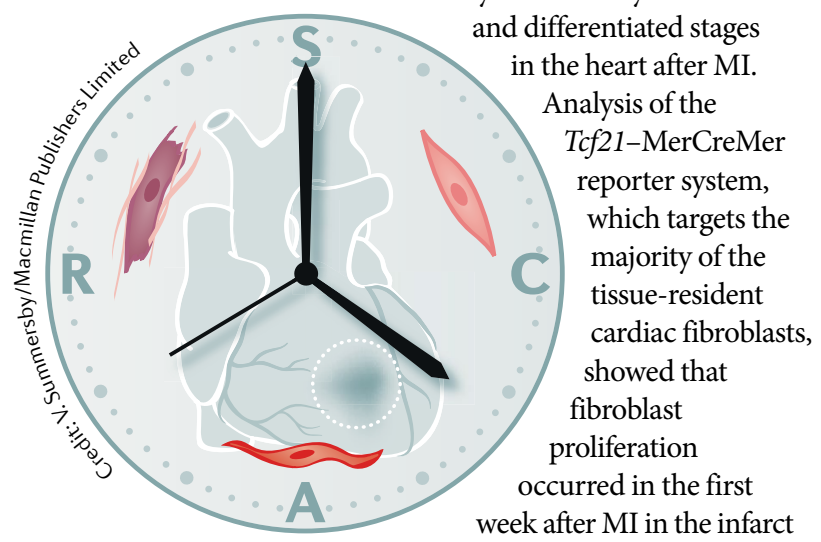

and border regions, reaching a peak of 3.5-fold expansion at 2-4 days compared with uninjured areas. The fibroblast content in injured regions persisted for weeks, suggesting that these new fibroblasts are robust and do not undergo cell turnover. Activated fibroblasts, labelled using the Postn-MerCreMer system, were shown to derive from Tcf21-traced cells 4 days after MI. Postn-traced cells had similar proliferation dynamics as Tcf21-labelled cells, expressed smooth muscle $\alpha$-actin (aSMA) - a myofibroblast marker encoded by Acta2 - at 4-7 days after MI, and showed no significant proliferation rates or aSMA expression 2 weeks after MI. Acta2-lineage tracing confirmed that myofibroblasts appear at day 3-4 after MI, and lose aSMA expression by 10 days after MI. Altogether, these findings demonstrate that myofibroblasts are not a long-lasting differentiated state of fibroblasts, and that fibroblasts transition to an alternate stage refractory to proliferation by 10-14 days after MI. Histological analysis of the scar also revealed that the loss of aSMA expression and myofibroblast phenotype preceded the reorganization of the ECM associated with scar maturation, suggesting that myofibroblasts are a transient cellular support network that preserves ventricular wall integrity before collagen accumulation.

mRNA profiling of $T c f 21$-lineage traced cells at different time points revealed that the greatest differences in gene expression between fibroblasts from infarcted hearts and fibroblasts from healthy hearts occurred 3 days after MI. Fibroblasts isolated 4 weeks after MI were the most related to the quiescent, uninjured fibroblast phenotype, but differentially expressed a unique panel of genes, including Comp, Chad, and Clip2, that is usually expressed by chondroblasts and osteoblasts. The investigators referred to this unique fibroblast phenotype as the matrifibrocyte. "We propose that the matrifibrocyte is a MI scar-specific differentiated state of the cardiac fibroblast that underlies the long-term stability of the fibrotic scar itself," explains Molkentin. "Our hopes are that better molecular characterization of the specific stages of fibroblast differentiation, along with an understanding of their nodal regulatory pathways, will permit better targeted therapies to control these cells in the heart," he concludes.

"This is a very well executed study that confirms the existing conceptual paradigm on the cell biological dynamics of fibroblast activation in the infarcted myocardium using robust new genetic tools," says Nikolaos Frangogiannis, who was not involved in the study. Frangogiannis explains that several questions remain unanswered, including whether different functional fibroblast subsets regulate different processes in the heart, such as ECM remodelling, inflammation, angiogenesis, or repair. "Answers to these questions are critical to design interventions that target detrimental effects of fibroblasts while preserving their reparative functions," he adds.

Alexandra Le Bras

ORIGINAL ARTICLE Fu, X. et al. Specialized fibroblast differentiated states underlie scar formation in the infarcted heart.J. Clin. Invest. https://doi.org/10.1172/JCl98215 (2018) FURTHER READING Tallquist, M. D. 8 Molkentin, J. D. Redefining the identity of cardiac fibroblasts. Nat. Rev. Cardiol. 14, 484-491 (2017) 